
\title{
25 Research Soure \\ The Effects of Cancer Survivor Support Service on Distress in South Korea: A Nationwide Prospective Pilot Study
}

Hyun Jeong Lee

National Cancer Center

Young Ae Kim ( $\square$ elkim7@gmail.com )

National Cancer Center https://orcid.org/0000-0002-3819-0028

Seong Yeob Ryu

Chonnam National University

Mison Chun

Ajou University

Chang-Yeol Yim

Chonbuk National University Medical School: Jeonbuk National University Medical School

Hee-Taik Kang

Chungbuk National University

Jung Hun Kang

Gyeongsang National University

Jung-Sik Huh

Jeju National University

Jong-Heun Kim

National Cancer Center

Kyu-Hyoung Lim

Kangwon National University

So Youn Jung

National Cancer Center

Hyoung-Cheol Kwon

Chonbuk National University: Jeonbuk National University

Eurah Goh

Kangwon National University

Yeon-Seung Lee

National Cancer Center

Hee Young Ju

Samsung Medical Center

E Hwa Yun 
National Cancer Center

Yoon Jung Chang

National Cancer Center

\section{Research article}

Keywords: cancer survivor, cancer survivorship management, distress, support service, government-led

Posted Date: July 28th, 2021

DOI: https://doi.org/10.21203/rs.3.rs-753682/v1

License: (9) This work is licensed under a Creative Commons Attribution 4.0 International License. Read Full License 


\section{Abstract}

Background: To investigate the characteristics of cancer survivors and the effects of the services of the Korean Cancer Survivorship Center Pilot Project launched by the South Korean government on distress.

Methods: A prospective observational cohort study was performed in cancer survivors who completed primary treatment. Cancer survivors' distress and symptoms such as fatigue, pain, depressive mood, anxiety, and insomnia were evaluated by well-trained nurses. Regarding to their needs, medical and psychosocial support services were provided.

Results: This study included 1,921 cancer survivors, with a mean age of 57.3 years ( $68.7 \%$ females). The breast cancer was most common, followed by stomach and colorectal cancer. Psychosocial and medical support decreased the percentage of the high-distress group from $50.9 \%$ to $30.5 \%$ and decreased the percentage of cancer survivors with high scores in fatigue, pain, anxiety, depressive mood, and insomnia. The independent predictors of a low distress level after the use of the services were older age, the relief of fatigue, pain, and insomnia.

Conclusions: This study showed that psychosocial and medical support is associated with the lower distress and physical and mental symptoms of cancer survivors. Psychosocial and medical support could contribute to distress relief in cancer survivors. Further management strategies for fatigue, pain, and insomnia are required.

\section{Background}

The numbers of cancer survivors are gradually increasing owing to advances in screening and treatment strategies. However, they still suffer from various difficulties including long-term treatment effects, lateeffects of treatment, distress, anxiety, uncertainty, fatigue, secondary malignancy, and other medical illness [1-5]. Unmanaged distress has negative effects on all-cause and cancer-related morbidity and mortality, as well as quality of life [6,7]. Distress is defined as 'an unpleasant experience of a mental, physical, social, or spiritual nature' [8]. Because distress is caused by physical, psychological, and social problems, psychosocial and medical support should be included in cancer care [9].

The 5-year relative survival rate of cancer patients in South Korea improved substantially from $41.2 \%$ for the period $1993-1995$ to $70.6 \%$ for the period 2012-2016; there were approximately 1,740,000 cancer survivors in 2016 [10]. The age-standardized 5-year net survival rates in South Korea are among the highest of the 32 countries in the Organization for Economic Cooperation and Development (OECD); 5year survival rates are $71.8 \%$ in colon cancer patients, $71.1 \%$ in rectal cancer patients, $68.9 \%$ in stomach cancer patients (ranked first among OECD countries) and $25.1 \%$ in lung cancer patients (ranked third among OECD countries) [11]. Nevertheless, South Korea has lacked a systematic support system for cancer survivors. To improve the health status of cancer survivors and facilitate their return to normal social lives, the Korean Ministry of Health and Welfare together with the National Cancer Center, a 
government-endorsed organization, launched the Korean Cancer Survivorship Center Pilot Project (KCSCP) in July 2017.

We investigated characteristics of cancer survivors visiting cancer survivorship centers (CSCs) and the associations of the Korean Cancer Survivorship Center Pilot Program with survivors' distress and distressrelated symptoms including pain, fatigue, anxiety, depressive mood, and insomnia.

\section{Methods}

\section{Study Design}

This study was a prospective, observational cohort study performed from July 2017 to December 2018 in South Korea. Seven regional cancer centers and one national cancer center covering the regional areas in South Korea were designated in 2017, and one more center was joined in 2018.

Before supportive services, well-trained nurses evaluate cancer survivors in various areas including distress, distress related symptoms, and social welfare area. After the initial assessment, survivors were divided into low-risk groups (all areas with low symptom burden) and high-risk groups (one or more of the eight areas with moderate to high symptom burden). Cancer survivors in the high-risk group were evaluated by doctors at CSC's cancer-survivorship clinic because of their greater symptom burdens and unmet needs for supportive care and referred to specialists in other medical departments as needed. Then, they were provided with cancer survivor support services (Supplementary Table 1 ) as needed. They were reassessed after 1 month or 3 months from baseline according to their risk levels using part of the initial assessment tool.

\section{Participants}

This program was targeted to any adult cancer survivor who completed curative-intent anti-cancer treatment including surgery, chemotherapy, or radiotherapy. Survivors undergoing maintenance hormonal therapy were also included. This study excluded individuals at the end-stage of cancer needing hospicepalliative care. Cancer survivors were recruited through a variety of channels, such as referrals from medical staff, recommendations from other patients, or advertisements. A total of 2,601 cancer survivors registered to the CSCs, however, 156 individuals refused to participate in this study; therefore, 2,445 subjects were finally included. Of these, 1,921 subjects completed re-assessment of distress and were included in the analyses. Informed consent was obtained from each study participant. The study was approved by the institutional review boards of National Cancer Center (IRB No.: NCC2017-0204) and other seven hospitals.

\section{Outcome Measures}


Tools included physical and mental health, and social welfare items. The baseline assessment tool comprised a self-report questionnaire and an observer-assessment tool used by nurses. We gathered data about basic sociodemographic factors (age, sex, education level, and marital status), income, life behavior (smoking and drinking habits), health conditions (body mass index, comorbidity), distress, and distress related symptoms including anxiety, depression, insomnia, fatigue, and pain by paper questionnaire and interview. We used patient treatment summaries to obtain clinical information related to cancer diagnosis and treatment.

We used the National Comprehensive Cancer Network (NCCN) distress thermometer (DT) and Problem list for distress screening, which were validated in Korean $[12,13]$. The DT was a one-item measure of distress - on a scale of zero to 10, 10 being the worst. A cut-off of $\geq 4$ has been accepted by the NCCN to indicate clinically meaningful distress [8]. Using this cut-off, cancer survivors were divided into high- and low-distress groups. For screening other domain including fatigue, pain, anxiety, depressive mood, and insomnia, we used 11-point $(0-10)$ Likert scales $[14,15]$. We used the EuroQuo-visual analog scale (EQVAS) to assess the quality of life $[16,17]$.

\section{Cancer Survivor Support Service}

A multidisciplinary cancer survivor support team comprising doctors, nurses, and social workers provided the cancer survivor support services. The CSC nurses who were oncology nurses or nurses with at least 2year experience in cancer care conducted individual education or counseling during the baseline assessment. The CSC social workers were medical social workers or social workers with at least 2-year experience in counseling in other institutions.

When the assessment score was 4 or more, we provided services by symptom categories. Primarily several educational programs of various topics, including management of adverse effects of cancer treatment, proper nutrition, undergoing secondary malignancy screening, the importance of vaccinations, and maintaining a healthy lifestyle such as quitting smoking or alcohol, managing distress, and ensuring good sleep routines were provided. In addition, counseling, group programs such as programs of exercise and psychological support, social welfare counseling or cancer survivorship clinics treatment were provided if needed. The composition of the program differed according to the conditions in each CSC.

\section{Statistical Analyses}

We analyzed the continuous variables using an independent t-test, and categorical variables using the chi-square test (or Fisher's exact test, if appropriate). We used the McNemar test to evaluate the changes in proportion of distress, and distress related symptoms including fatigue, pain, anxiety, depressive mood, and insomnia. Multivariate analysis of covariance was used to examine estimated mean scores in EQVAS, distress, pain, fatigue, anxiety, depressive mood, and insomnia according to distress levels after adjusting for age, sex, education levels, economic status, cancer diagnosis, cancer treatment, and time 
from diagnosis to CSC visit. Univariate logistic regression was carried out, and significant variables were entered into multivariate logistic regression to determine predictors of a low-distress level after services. Statistical analyses were performed using SAS (version 9.4, SAS Inc., Cary, NC, USA). A two-sided P-value $<0.05$ was considered to indicate significance.

\section{Results}

\section{Baseline characteristics}

This study included 1,921 cancer survivors, with a mean age of 57.3 years ( $68.7 \%$ females). The most common cancer was breast cancer $(41.3 \%)$, followed by stomach cancer $(21.7 \%)$, colorectal cancer (11.5\%), and other cancers. Cancer survivors were grouped by their DT scores; $49.1 \%$ had low distress (DT score $<4$ ) and $50.9 \%$ had high distress (DT score $\geq 4$ ). Compared to the low-distress group, the highdistress group were younger and had a higher percentage of the high-risk groups in terms of symptom burdens at baseline, being female, having breast cancer, and undergoing non-surgical treatment modalities (chemotherapy, radiotherapy, or other anti-cancer treatment). Conversely, the high-distress group had a lower percentage of patients with stomach or colorectal cancers, compared to the lowdistress group (Table 1). 
Table 1

Characteristics of cancer survivors according to distress level at baseline

\begin{tabular}{|c|c|c|c|c|c|c|c|}
\hline \multirow[t]{3}{*}{ Variables } & \multirow{2}{*}{\multicolumn{2}{|c|}{$\begin{array}{l}\text { Total } \\
n=1,921\end{array}$}} & \multirow{2}{*}{\multicolumn{2}{|c|}{$\begin{array}{l}\text { Low-distress } \\
\text { group }^{\dagger} \\
n=943 \\
(49.1 \%)\end{array}$}} & \multirow{2}{*}{\multicolumn{2}{|c|}{$\begin{array}{l}\text { High-distress } \\
\text { group }^{\ddagger} \\
n=978 \\
(50.9 \%)\end{array}$}} & \multirow[t]{3}{*}{$\begin{array}{l}p- \\
\text { value }\end{array}$} \\
\hline & & & & & & & \\
\hline & $\mathrm{n}$ & (\%) & $\mathbf{n}$ & $(\%)$ & $\mathbf{n}$ & $(\%)$ & \\
\hline Age, year & \multicolumn{2}{|c|}{$57.3 \pm 11.2$} & \multicolumn{2}{|c|}{$58.8 \pm 10.9$} & \multicolumn{2}{|c|}{$55.9 \pm 11.2$} & $\begin{array}{l}< \\
0.001^{* *}\end{array}$ \\
\hline Female & 1320 & $(68.7)$ & 603 & $(63.9)$ & 717 & (73.3) & $\begin{array}{l}< \\
0.001^{\star *}\end{array}$ \\
\hline \multicolumn{8}{|l|}{ Education } \\
\hline$<$ High school & 563 & $(29.3)$ & 294 & $(31.2)$ & 269 & $(27.5)$ & 0.077 \\
\hline High school & 702 & $(36.5)$ & 342 & $(36.3)$ & 360 & $(36.8)$ & 0.805 \\
\hline$>$ High school & 635 & $(33.1)$ & 299 & (31.7) & 336 & $(34.4)$ & 0.217 \\
\hline \multicolumn{8}{|l|}{ Income, dollars/month } \\
\hline$<850$ & 395 & $(21.9)$ & 183 & $(20.7)$ & 212 & $(23.0)$ & 0.244 \\
\hline $850 \sim 1,700$ & 319 & $(16.6)$ & 154 & $(16.3)$ & 165 & $(16.9)$ & 0.750 \\
\hline $1,700 \sim 2,550$ & 333 & $(17.3)$ & 163 & $(17.3)$ & 170 & $(17.4)$ & 0.955 \\
\hline $2,550 \sim 3,400$ & 304 & $(15.8)$ & 160 & $(17.0)$ & 144 & $(14.7)$ & 0.178 \\
\hline$\geq 3,400$ & 456 & $(23.7)$ & 224 & $(23.8)$ & 232 & $(23.7)$ & 0.987 \\
\hline Married/living with partner & 1,577 & $(82.9)$ & 789 & $(84.2)$ & 788 & $(81.6)$ & 0.128 \\
\hline With jobs & 644 & $(33.6)$ & 329 & $(34.9)$ & 315 & $(32.4)$ & 0.244 \\
\hline Time from cancer diagnosis, years & $3.4 \pm 3$ & & $3.6 \pm$ & & $3.1=$ & & $0.003^{*}$ \\
\hline \multicolumn{8}{|l|}{ Cancer diagnosis } \\
\hline Breast cancer & 792 & $(41.3)$ & 359 & $(38.2)$ & 433 & $(44.4)$ & $0.005^{*}$ \\
\hline Stomach cancer & 415 & $(21.7)$ & 227 & $(24.1)$ & 188 & $(19.3)$ & $0.010^{*}$ \\
\hline Colorectal cancer & 221 & $(11.5)$ & 124 & $(13.2)$ & 97 & $(9.9)$ & $0.027^{*}$ \\
\hline Other cancer & 488 & $(25.5)$ & 231 & $(24.5)$ & 257 & $(26.4)$ & 0.363 \\
\hline \multicolumn{8}{|l|}{ Cancer treatment } \\
\hline Surgery & 1,816 & (95.8) & 890 & (95.4) & 926 & $(96.2)$ & 0.406 \\
\hline
\end{tabular}




\begin{tabular}{|c|c|c|c|c|c|c|c|}
\hline \multirow[t]{3}{*}{ Variables } & \multirow{2}{*}{\multicolumn{2}{|c|}{$\begin{array}{l}\text { Total } \\
n=1,921\end{array}$}} & \multirow{2}{*}{\multicolumn{2}{|c|}{$\begin{array}{l}\text { Low-distress } \\
\text { group }^{\dagger} \\
n=943 \\
(49.1 \%)\end{array}$}} & \multirow{2}{*}{\multicolumn{2}{|c|}{$\begin{array}{l}\text { High-distress } \\
\text { group }^{\ddagger} \\
n=978 \\
(50.9 \%)\end{array}$}} & \multirow[t]{3}{*}{$\begin{array}{l}p- \\
\text { value }\end{array}$} \\
\hline & & & & & & & \\
\hline & $\mathbf{n}$ & (\%) & $\mathbf{n}$ & $(\%)$ & $\mathbf{n}$ & $(\%)$ & \\
\hline Chemotherapy & 973 & $(51.3)$ & 454 & $(48.7)$ & 519 & $(54.0)$ & $0.021^{*}$ \\
\hline Radiotherapy & 895 & $(47.2)$ & 411 & $(44.1)$ & 484 & $(50.3)$ & $0.007^{*}$ \\
\hline $\begin{array}{l}\text { Other cancer treatment } \\
\text { (hormone therapy, etc) }\end{array}$ & 515 & $(27.4)$ & 223 & $(24.1)$ & 292 & $(30.6)$ & $0.002^{*}$ \\
\hline \multicolumn{8}{|l|}{ CSC visit route } \\
\hline $\begin{array}{l}\text { Recommendation of hospital } \\
\text { staff }\end{array}$ & 639 & $(33.3)$ & 358 & $(38.0)$ & 281 & $(28.7)$ & $\begin{array}{l}< \\
0.001^{* *}\end{array}$ \\
\hline Recommendation of oncologist & 365 & $(19.0)$ & 210 & $(22.3)$ & 155 & $(15.8)$ & $0.001^{\star \star}$ \\
\hline $\begin{array}{l}\text { In-hospital advertising } \\
\text { (poster, leaflet, banner, etc.) }\end{array}$ & 339 & $(17.6)$ & 117 & $(12.4)$ & 222 & $(22.7)$ & $<.001^{\star *}$ \\
\hline $\begin{array}{l}\text { Recommendation from other } \\
\text { patients }\end{array}$ & 159 & $(8.3)$ & 68 & $(7.2)$ & 91 & $(9.3)$ & 0.096 \\
\hline $\begin{array}{l}\text { Recommendation from family } \\
\text { and friends }\end{array}$ & 77 & $(4.0)$ & 38 & $(4.0)$ & 39 & $(4.0)$ & 0.963 \\
\hline Advertising media & 36 & $(1.9)$ & 18 & $(1.9)$ & 18 & $(1.8)$ & 0.912 \\
\hline Other & 136 & $(7.1)$ & 68 & $(7.2)$ & 68 & $(7.0)$ & 0.826 \\
\hline \multicolumn{8}{|l|}{ Scores at baseline } \\
\hline EQ-VAS & \multicolumn{2}{|c|}{$67.4 \pm 18.0$} & \multicolumn{2}{|c|}{$72.8 \pm 16.9$} & \multicolumn{2}{|c|}{$62.1 \pm 17.5$} & $0.001^{\star \star}$ \\
\hline Distress & \multicolumn{2}{|c|}{$4.0 \pm 2.5$} & \multicolumn{2}{|c|}{$1.9 \pm 1.1$} & \multicolumn{2}{|c|}{$6.0 \pm 1.6$} & $<.001^{* *}$ \\
\hline Fatigue & \multicolumn{2}{|c|}{$4.2 \pm 2.6$} & \multicolumn{2}{|c|}{$3.1 \pm 2.2$} & \multicolumn{2}{|c|}{$5.3 \pm 2.5$} & $<.001^{\star \star}$ \\
\hline Pain & \multicolumn{2}{|c|}{$2.4 \pm 2.7$} & \multicolumn{2}{|c|}{$1.6 \pm 2.1$} & \multicolumn{2}{|c|}{$3.2 \pm 2.9$} & $<.001^{\star *}$ \\
\hline Anxiety & \multicolumn{2}{|c|}{$2.8 \pm 2.7$} & \multicolumn{2}{|c|}{$1.5 \pm 1.9$} & \multicolumn{2}{|c|}{$4.0 \pm 2.8$} & $\begin{array}{l}< \\
0.001^{\star *}\end{array}$ \\
\hline
\end{tabular}




\begin{tabular}{|c|c|c|c|c|c|c|}
\hline \multirow[t]{3}{*}{ Variables } & \multirow{2}{*}{\multicolumn{2}{|c|}{$\begin{array}{l}\text { Total } \\
n=1,921\end{array}$}} & \multirow{2}{*}{\multicolumn{2}{|c|}{$\begin{array}{l}\text { Low-distress } \\
\text { group }^{\dagger} \\
n=943 \\
(49.1 \%)\end{array}$}} & \multirow{2}{*}{$\begin{array}{l}\text { High-distress } \\
\text { group }^{\ddagger} \\
n=978 \\
(50.9 \%)\end{array}$} & \multirow[t]{3}{*}{$\begin{array}{l}p- \\
\text { value }\end{array}$} \\
\hline & & & & & & \\
\hline & $\mathbf{n}$ & (\%) & $\mathrm{n}$ & $(\%)$ & $(\%)$ & \\
\hline Depressive mood & \multicolumn{2}{|c|}{$2.5 \pm 2.7$} & \multicolumn{2}{|c|}{$1.1 \pm 1.6$} & $3.8 \pm 2.9$ & $\begin{array}{l}< \\
0.001^{* *}\end{array}$ \\
\hline Insomnia & \multicolumn{2}{|c|}{$2.8 \pm 2.9$} & \multicolumn{2}{|c|}{$1.8 \pm 2.3$} & $3.8 \pm 3.1$ & $\begin{array}{l}< \\
0.001^{* *}\end{array}$ \\
\hline High-risk group§ & 1,475 & $(76.8)$ & 622 & $(66.0)$ & $853 \quad(87.2)$ & $\begin{array}{l}< \\
0.001^{\star *}\end{array}$ \\
\hline \multicolumn{7}{|c|}{$\begin{array}{l}{ }^{\dagger} \text { Low-distress group had distress thermometer (DT) scores }<4 ;{ }^{\ddagger} H \text { High-distress group had DT scores } \geq \\
4 ; \text { \$The high-risk group had moderate to high symptom burdens in one or more areas among eight } \\
\text { areas (nutrition and diet, fatigue, pain, anxiety, depressive mood, insomnia, exercise, and social } \\
\text { welfare) }\end{array}$} \\
\hline \multicolumn{7}{|c|}{${ }^{*} p$ value $<0.05,{ }^{* *} p$ value $<0.001$, CSC, Cancer Survivorship Center } \\
\hline
\end{tabular}

Changes of proportion of distress and distress related symptoms after using the cancer survivor support services

Cancer survivors receiving support services decreased the proportion of people with high symptoms scores (4 point or more), such as distress $(978,50.9 \%$ vs $586,30.5 \%)$, fatigue $(1,078,59.2 \%$ vs. 726 , $39.8 \%$ ), pain ( $567,31.6 \%$ vs. $380,21.2 \%)$, anxiety $(678,37.5 \%$ vs. $322,17.8 \%)$, depressive mood (571, $31.7 \%$ vs. $27515.2 \%)$, and insomnia (682, 37.6\% vs. 409, 22.5\%), as assessed after 1 or 3 months compared to baseline (all $p<0.001$ ) (Fig. 1).

\section{Characteristics of cancer survivors according to distress levels after using cancer survivor support services}

Table 2 shows demographic and clinical characteristics in low- and high-distress group after cancer survivor support services. Compared with the low-distress group after receiving the service, the highdistress group was younger, the proportion of male, those with a monthly income of 2,550 3,400 dollars, those with high school graduates and below were low, and the proportion of those with a monthly income of $\geq 3,400$ dollars was high. The proportion of stomach and colorectal cancer survivors in the lowdistress group was higher. The proportion of breast cancer survivors was higher in the high-distress group, and the proportions of survivors who received chemotherapy, radiotherapy, and other cancer treatment (e.g., hormonal therapy, targeted therapy, endoscopic mucosal resection, radioactive iodine therapy, etc.) was higher. The high-distress group had lower quality of life and higher fatigue, pain, 
anxiety, depressive mood, and insomnia than the low-distress group, after adjusting for age, sex, education levels, economic status, cancer diagnosis, cancer treatment and time from diagnosis to visit. 
Table 2

Characteristics of cancer survivors according to distress levels after using cancer survivor support services

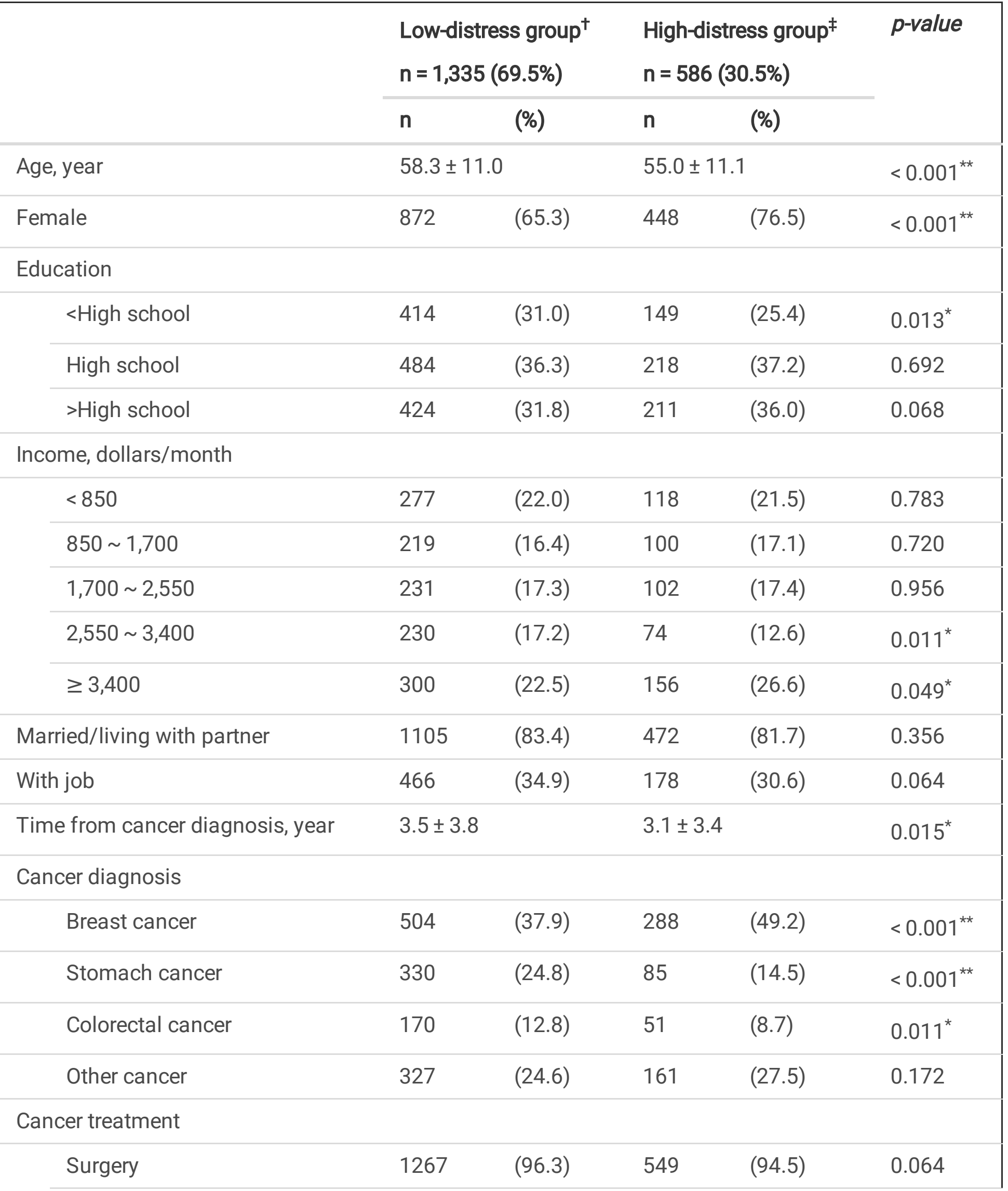




\begin{tabular}{|c|c|c|c|c|c|}
\hline & \multicolumn{2}{|c|}{$\begin{array}{l}\text { Low-distress group }^{\dagger} \\
n=1,335(69.5 \%)\end{array}$} & \multicolumn{2}{|c|}{$\begin{array}{l}\text { High-distress group }{ }^{\ddagger} \\
n=586(30.5 \%)\end{array}$} & \multirow[t]{2}{*}{ p-value } \\
\hline & $\mathbf{n}$ & $(\%)$ & $\mathrm{n}$ & $(\%)$ & \\
\hline Chemotherapy & 645 & $(49.0)$ & 328 & $(56.6)$ & $0.003^{*}$ \\
\hline Radiotherapy & 570 & $(43.4)$ & 325 & $(55.8)$ & $<0.001^{\star *}$ \\
\hline Other cancer treatment & 323 & $(24.7)$ & 192 & (33.5) & $<0.001^{\star \star}$ \\
\hline \multicolumn{6}{|l|}{ Scores after services§ } \\
\hline EQ-VAS & $78.7 \pm 0.9$ & & $66.9 \pm 1.0$ & & $<0.001^{\star \star}$ \\
\hline Distress & $1.8 \pm 0.1$ & & $5.1 \pm 0.1$ & & $<0.001^{\star \star}$ \\
\hline Fatigue & $2.7 \pm 0.1$ & & $4.8 \pm 0.1$ & & $<0.001^{* *}$ \\
\hline Pain & $1.1 \pm 0.1$ & & $3.0 \pm 0.2$ & & $<0.001^{\star \star}$ \\
\hline Anxiety & $1.3 \pm 0.1$ & & $3.2 \pm 0.2$ & & $<0.001^{\star \star}$ \\
\hline Depressive mood & $1.3 \pm 0.1$ & & $3.1 \pm 0.1$ & & $<0.001^{\star *}$ \\
\hline Insomnia & $0.9 \pm 0.1$ & & $2.8 \pm 0.1$ & & $<0.001^{* *}$ \\
\hline \multicolumn{6}{|c|}{$\begin{array}{l}{ }^{\dagger} \text { Low-distress group had distress thermometer (DT) score }<4 ;{ }^{\ddagger} \text { High-distress group had DT scores } \geq 4 \\
\text { \$MANCOVA, estimated mean } \pm \text { standard error; adjusted for age, sex, education levels, economic } \\
\text { status, cancer diagnosis, cancer treatment and time from diagnosis to visit } \\
{ }^{*} p \text { value }<0.05,{ }^{* *} p \text { value }<0.001 \\
\text { EQ-VAS, EuroQuo-visual analog scale }\end{array}$} \\
\hline
\end{tabular}

\section{Predictors Of Low Distress Level After Using The Services}

Univariate analyses revealed that older age, male, a lower education level, longer length of time from cancer diagnosis, stomach, and colorectal cancer (vs. breast cancer), no history of chemotherapy, radiotherapy, or other anti-cancer treatment, and improvement of fatigue, pain, anxiety, and insomnia were associated with a low distress level after services. Multivariate logistic regression analysis showed that after using services, older age and improvement of fatigue, pain, and insomnia were independently associated with a low distress level (Table 3). 
Table 3

Predicting factors of a low distress level after services

Cancer-survivor characteristics
Univariate analysis

\begin{tabular}{|c|c|c|c|c|c|}
\hline OR & $95 \% \mathrm{Cl}$ & $\begin{array}{l}p- \\
\text { value }\end{array}$ & OR & $95 \% \mathrm{Cl}$ & $\begin{array}{l}p- \\
\text { value }\end{array}$ \\
\hline 1.028 & $\begin{array}{l}1.019- \\
1.037\end{array}$ & $\begin{array}{l}< \\
0.001^{* *}\end{array}$ & 1.023 & $\begin{array}{l}1.010- \\
1.037\end{array}$ & $\begin{array}{l}<.001^{*} \\
\end{array}$ \\
\hline 0.580 & $\begin{array}{l}0.465- \\
0.724\end{array}$ & $\begin{array}{l}<.001^{\star *} \\
0\end{array}$ & 0.825 & $\begin{array}{l}0.598- \\
1.137\end{array}$ & 0.240 \\
\hline 0.853 & $\begin{array}{l}0.754- \\
0.965\end{array}$ & $0.012^{*}$ & 1.042 & $\begin{array}{l}0.888- \\
1.222\end{array}$ & 0.615 \\
\hline
\end{tabular}

Age, year

Female $($ ref $=$ male $)$

Education level

Income

Cancer survivors with jobs $($ ref $=$ without jobs)

Married/living with partner (ref = single/divorced, widowed)

Time from cancer diagnosis, year

Cancer

diagnosis

$$
\text { diagnosis }
$$

\begin{tabular}{|c|c|c|c|c|c|c|c|}
\hline & Stomach cancer & 2.218 & $\begin{array}{l}1.679- \\
2.932\end{array}$ & $\begin{array}{l}< \\
0.001^{*}\end{array}$ & 1.235 & $\begin{array}{l}0.775- \\
1.967\end{array}$ & 0.374 \\
\hline & Colorectal cancer & 1.905 & $\begin{array}{l}1.349- \\
2.689\end{array}$ & $\begin{array}{l}< \\
0.001^{*}\end{array}$ & 1.183 & $\begin{array}{l}0.736- \\
1.900\end{array}$ & 0.487 \\
\hline & Other cancer & 1.161 & $\begin{array}{l}0.915- \\
1.472\end{array}$ & 0.220 & 0.811 & $\begin{array}{l}0.573- \\
1.149\end{array}$ & 0.239 \\
\hline \multirow[t]{4}{*}{$\begin{array}{l}\text { Cancer } \\
\text { treatment }\end{array}$} & $\begin{array}{l}\text { Surgery (ref = no } \\
\text { surgery) }\end{array}$ & 1.539 & $\begin{array}{l}0.973- \\
2.433\end{array}$ & 0.065 & & & \\
\hline & $\begin{array}{l}\text { Chemotherapy (ref } \\
=\text { no } \\
\text { chemotherapy) }\end{array}$ & 0.740 & $\begin{array}{l}0.608- \\
0.900\end{array}$ & $0.003^{*}$ & 0.796 & $\begin{array}{l}0.627- \\
1.010\end{array}$ & 0.060 \\
\hline & $\begin{array}{l}\text { Radiotherapy (ref = } \\
\text { no radiotherapy) }\end{array}$ & 0.606 & $\begin{array}{l}0.498- \\
0.737\end{array}$ & $\begin{array}{l}< \\
0.001^{*}\end{array}$ & 0.846 & $\begin{array}{l}0.624- \\
1.148\end{array}$ & 0.284 \\
\hline & $\begin{array}{l}\text { Other treatment } \\
\text { (ref = no other } \\
\text { treatment) }\end{array}$ & 0.650 & $\begin{array}{l}0.525- \\
0.805\end{array}$ & $\begin{array}{l}< \\
0.001^{*}\end{array}$ & 0.898 & $\begin{array}{l}0.685- \\
1.178\end{array}$ & 0.437 \\
\hline
\end{tabular}




\begin{tabular}{|c|c|c|c|c|c|c|c|}
\hline \multicolumn{2}{|c|}{ Cancer-survivor characteristics } & \multicolumn{3}{|c|}{ Univariate analysis } & \multicolumn{3}{|c|}{ Multivariate analysis } \\
\hline & & OR & $95 \% \mathrm{Cl}$ & $\begin{array}{l}p- \\
\text { value }\end{array}$ & OR & $95 \% \mathrm{Cl}$ & $\begin{array}{l}p- \\
\text { value }\end{array}$ \\
\hline \multirow{5}{*}{$\begin{array}{l}\text { Change of } \\
\text { scores from } \\
\text { baseline }\end{array}$} & $\Delta$ Fatigue & 1.163 & $\begin{array}{l}1.109- \\
1.220\end{array}$ & $\begin{array}{l}< \\
0.001^{*}\end{array}$ & 1.124 & $\begin{array}{l}1.064- \\
1.187\end{array}$ & $\begin{array}{l}< \\
0.001^{* *}\end{array}$ \\
\hline & $\Delta$ Pain & 1.122 & $\begin{array}{l}1.066- \\
1.181\end{array}$ & $\begin{array}{l}< \\
0.001^{*}\end{array}$ & 1.065 & $\begin{array}{l}1.004- \\
1.129\end{array}$ & $0.036^{*}$ \\
\hline & $\Delta$ Anxiety & 1.054 & $\begin{array}{l}1.005- \\
1.104\end{array}$ & $0.029^{*}$ & 0.996 & $\begin{array}{l}0.943- \\
1.052\end{array}$ & 0.881 \\
\hline & $\Delta$ Depressive mood & 1.039 & $\begin{array}{l}0.991- \\
1.089\end{array}$ & 0.114 & & & \\
\hline & $\Delta$ Insomnia & 1.112 & $\begin{array}{l}1.063- \\
1.164\end{array}$ & $\begin{array}{l}< \\
0.001^{*}\end{array}$ & 1.079 & $\begin{array}{l}1.024- \\
1.137\end{array}$ & $0.004^{*}$ \\
\hline \multicolumn{8}{|c|}{${ }^{*} p$ value $<0.05,{ }^{* *} p$ value $<0.001$} \\
\hline \multicolumn{8}{|c|}{ OR, Odds ratio; $\mathrm{Cl}$, confidence interval; ref, reference } \\
\hline
\end{tabular}

\section{Discussion}

This study showed that using the cancer survivor support services was related to amelioration of the distress and symptoms such as fatigue, pain, anxiety, depressive mood, and insomnia in cancer survivors. Our multidisciplinary cancer survivor support services include education, counseling, programs of exercise, or psychological support and cancer survivorship clinics, if needed. Previous studies showed that psychoeducational intervention, exercise, and education programs could improve symptom clusters including fatigue, depression, insomnia, pain, functional performance, and quality of life [18].

Within this study population, $75 \%$ were stomach, colorectal or breast cancer survivors, reflecting the current cancer epidemiological profile and clinical outcomes. In South Korea, the most common malignancy is stomach cancer $(13.3 \%)$, followed by colorectal cancer $(12.3 \%)$; breast cancer is the fifth most common cancer $(9.5 \%)$ in the entire population and the most common cancer among females (19.9\%). Furthermore, these three cancers have high 5-year survival rates $(76.0,75.9$, and $92.7 \%$, respectively) [10].

Younger cancer survivors experienced more distress levels at baseline and were not likely to improve the distress after using cancer survivor support services. A previous study [19] implied that younger patients may suffer a higher level of distress [19], perhaps due to economic vulnerability from decreased income, the larger disruption of social and familial roles in earlier developmental stages [20], a higher fear of progression, and more aggressive anti-cancer treatments [21]. In a previous study, younger survivors also reported more pain impact and intensity than older survivors [22]. 
At baseline, the high-distress group had high female predominance, with breast cancer survivors accounting for about half of this population. The need for supportive care among breast cancer survivors is relatively high [23]. Chemotherapy, radiotherapy, and long-term hormone therapy are widely applied to reduce the risks of breast cancer recurrence [24]. In particular, the many physical and mental symptoms caused by hormone therapy, e.g., hot flushes, night sweats, arthralgia, osteoporosis, insomnia, and depression [25], may lead to higher distress in survivors.

Older age, greater improvement of fatigue, pain and insomnia were independently associated with a low distress level after using the services in this study. Since our services focused on the general problem of cancer survivors and most cancer survivors visiting the CSC were middle-aged or old, specialized services for young cancer survivors are lacking. Therefore, they were unlikely to improve their distress after using support services.

Cancer-related fatigue is a common and distressing adverse effect of cancer and cancer therapy [26, 27]; fatigue is a common barrier for returning to work and the activities of daily life [28, 29]. Fatigue and distress have reciprocal effects on each other [30]. A study of prostate cancer survivors showed that the risk of distress was positively associated with fatigue, insomnia, urinary, bowel and androgen deprivation therapy-related symptoms, and financial difficulties [31]. Pain is also a common problem in cancer survivors [32]. Insomnia is related to fatigue itself. Insomnia contributes to additional risk for persistent fatigue after cancer treatment [33]. However, from a recent study [34], cognitive behavioral therapy did not significantly improve fatigue, although it could improve sleep.

Our project has several strengths. First, to the best of our knowledge, this pilot project is the first government-led national cancer survivor management program with adequate follow-up, worldwide. Our cancer survivorship management program is government-funded and based in academic cancer centers that were designated as regional cancer centers by the government to reduce the regional inequality of cancer care. South Koreans have good access to medical services. However, due to the lack of support systems for health and social welfare beyond the medical treatment of cancer survivors, this project was designed to develop and disseminate national programs, and to develop and operate services according to regional characteristics. Several countries have other cancer survivorship management models. For example, cancer survivorship management in the United States of America is conducted mainly by academic cancer centers. In the United Kingdom, the survivorship care model is based on the countrywide healthcare system, although medical care and other supportive services are provided by communitybased primary-care clinics, private institutions, or cancer charities [35]. In Australia, medical care and other supportive services for cancer survivors are provided by cancer centers in public hospitals, in part supported by the state government and private foundation. Their supportive services include providing information to cancer survivors and training for healthcare professionals [36]. Compared to previous studies, the advantage of our study was that it provided integrated support services to cancer survivors that manage various biopsychosocial issues and include psychosocial and medical support. 
This study had several limitations. First, selection bias might have occurred, in terms that those who were unable to visit a center during the daytime could not participate in this project. Second, the intervention services were diverse according to centers, and not standardized, making a quantitative assessment of their efficacies difficult. Further study designs should include standardized services. Third, primary due to lack of control group, our study result might be interpreted, considering the possibility that some people might experience self-remission even without any interventions.

\section{Conclusion}

This study is the first to report a nationwide government-led cancer survivor management program. Our pilot study showed positive effects on physical and mental health in cancer survivors. Predictors of a lowdistress level after utilization of services were older age, and greater improvement in symptoms of fatigue, pain, and insomnia. Specialized support services to manage major issues for younger cancer survivors, i.e., childbearing, childcare, marriage, study, or career, are needed. Furthermore, management strategies to control fatigue, pain and insomnia are also required.

\section{Abbreviations}

$\mathrm{Cl}$ confidence interval; CSC:cancer survivorship center; DT:distress thermometer; EQ-VAS:EuroQuo-visual analog scale; K-CSCP:Korean Cancer Survivorship Center Pilot Project; NCCN:National Comprehensive Cancer Network; OECD:Organization for Economic Cooperation and Development; OR:odds ratio; ref:reference.

\section{Declarations}

\section{Acknowledgements}

We would like to thank Textcheck (www.textcheck.com) for editing and reviewing this manuscript for English language.

\section{Authors' contributions}

HJL: Conceptualization, Methodology, Formal analysis, Writing - Original Draft, Writing - Review \& Editing YAK: Conceptualization, Methodology, Writing - Review \& Editing, Supervision, Project administration SYR, MC, CYY, HTK, JHK1, JSH, JHK2, KHL, SYJ, HCK, EG, YSL, and EHY: Data Curation HYJ: Methodology YJC: Supervision, Project administration

All authors have read and approved the manuscript.

\section{Funding}

None. 


\section{Availability of data and materials}

The datasets generated during and/or analysed during the current study are available from the corresponding author on reasonable request.

\section{Ethics approval and consent to participate}

This study was performed in line with the principles of the Declaration of Helsinki. The study was approved by the institutional review boards of National Cancer Center (IRB No.: NCC2017-0204) and other seven hospitals. Informed consent was obtained from each study participant due to the retrospective design.

\section{Consent for publication}

Not applicable.

\section{Competing interests}

The authors declare that they have no known competing financial interests or personal relationships that could have appeared to influence the work reported in this paper.

\section{Author details}

${ }^{1}$ Division of Cancer Control \& Policy, National Cancer Center, Goyang, Korea

${ }^{2}$ National Cancer Survivorship Center, National Cancer Center, Goyang, Korea

${ }^{3}$ Department of Psychiatry and Behavioral Science, National Cancer Center, Goyang, Korea

${ }^{4}$ Department of Psychiatry, Seoul National University, Seoul, Korea

${ }^{5}$ Department of Surgery, Chonnam National University Medical School, Gwangju, Korea

${ }^{6}$ Department of Radiation Oncology, Ajou University School of Medicine, Suwon, Korea

${ }^{7}$ Department of Internal Medicine, Chonbuk National University Medical School and Research Institute of Clinical Medicine of Chonbuk National University, Jeonju, Korea

${ }^{8}$ Biomedical Research Institute of Chonbuk National University Hospital, Jeonju, Korea

${ }^{9}$ Department of Family Medicine, Chungbuk National University Hospital, Cheongju, Korea

${ }^{10}$ Department of Family Medicine, Chungbuk National University College of Medicine, Cheongju, Korea 
${ }^{11}$ Division of Hematology-Oncology Department of Internal Medicine, College of Medicine, Gyeong-Sang National University, Jinju, Korea

${ }^{12}$ Department of Medicine, Gyeong-sang National University Hospital, Jinju, Korea

${ }^{13}$ Department of Urology, School of Medicine, Jeju National University, Jeju, Korea

${ }^{14}$ Department of Internal Medicine, Kangwon National University Hospital, Kangwon National University School of Medicine, Chuncheon, Korea

${ }^{15}$ Breast Cancer Center, National Cancer Center, Goyang, Korea

${ }^{16}$ Department of Radiation Oncology, Chonbuk National University Hospital, Jeonju, Korea

${ }^{17}$ Institute of Clinical Medicine of Chonbuk National University-Biomedical Research Institute, Chonbuk National University Hospital, Jeonju, Korea

${ }^{18}$ Department of Radiation Oncology, Chonbuk National University Medical School, Jeonju, Korea

${ }^{19}$ Department of Family Medicine, Postgraduate Collage of Medicine, Kangwon National University, Chuncheon, Korea

${ }^{20}$ Cancer Survivorship Branch, National Cancer Center, Goyang, Korea

${ }^{21}$ Department of Pediatrics, Samsung Medical Center, Seoul, Korea

${ }^{22}$ Division of Cancer Registration \& Surveillance, National Cancer Center, Goyang, Korea

${ }^{23}$ Center for Cancer Prevention \& Detection, National Cancer Center, Goyang, Korea

${ }^{24}$ National Hospice Center, National Cancer Center, Goyang, Korea

${ }^{25}$ Department of Cancer Control and Population Health, National Cancer Center Graduate School of Cancer Science and Policy, Goyang, Korea

\section{References}

1. Bower JE, Bak K, Berger A, Breitbart W, Escalante CP, Ganz PA, et al. Screening, assessment, and management of fatigue in adult survivors of cancer: an American Society of Clinical oncology clinical practice guideline adaptation. J Clin Oncol. 2014;32(17):1840-50.

2. Armenian SH, Lacchetti C, Lenihan D. Prevention and Monitoring of Cardiac Dysfunction in Survivors of Adult Cancers: American Society of Clinical Oncology Clinical Practice Guideline Summary. J Oncol Pract. 2017;13(4):270-5. 
3. Knobf MT. Clinical update: psychosocial responses in breast cancer survivors. Semin Oncol Nurs. 2011;27(3):e1-14.

4. Radivoyevitch T, Sachs RK, Gale RP, Molenaar RJ, Brenner DJ, Hill BT, et al. Defining AML and MDS second cancer risk dynamics after diagnoses of first cancers treated or not with radiation. Leukemia. 2016;30(2):285-94.

5. Casco S, Soto-Vega E. Development of Metabolic Syndrome Associated to Cancer Therapy: Review. Horm Cancer. 2016;7(5-6):289-95.

6. Batty GD, Russ TC, Stamatakis E, Kivimaki M. Psychological distress in relation to site specific cancer mortality: pooling of unpublished data from 16 prospective cohort studies. BMJ. 2017;356:j108.

7. Massie MJ. Prevalence of depression in patients with cancer. J Natl Cancer Inst Monogr. 2004. doi:10.1093/jncimonographs/lgh014.(32):57-71.

8. Holland JC, Bultz BD. National comprehensive Cancer N. The NCCN guideline for distress management: a case for making distress the sixth vital sign. J Natl Compr Canc Netw. 2007;5(1):37.

9. Admiraal JM, Hoekstra-Weebers J, Schroder CP, Tuinier W, Hospers GAP, Reyners AKL. Distress, problems, referral wish, and supportive health care use in breast cancer survivors beyond the first year after chemotherapy completion. Support Care Cancer. 2019. doi:10.1007/s00520-019-05030-6.

10. Jung KW, Won YJ, Kong HJ, Lee ES. Cancer Statistics in Korea: Incidence, Mortality, Survival, and Prevalence in 2016. Cancer Res Treat. 2019;51(2):417-30.

11. OECD. Health at a Glance 2019: OECD Indicators. Paris: OECD Publishing; 2019.

12. Shim EJ, Shin YW, Jeon HJ, Hahm BJ. Distress and its correlates in Korean cancer patients: pilot use of the distress thermometer and the problem list. Psychooncology. 2008;17(6):548-55.

13. Network NCC. NCCN Clinical Practice Guidelines in Oncology (NCCN Guidelines) for Distress Management 2015;V.1.2015.

14. Mitchell AJ, Baker-Glenn EA, Park B, Granger L, Symonds P. Can the Distress Thermometer be improved by additional mood domains? Part II. What is the optimal combination of Emotion Thermometers? Psychooncology. 2010;19(2):134-40.

15. Shim EJ, Hahm BJ, Yu ES, Kim HK, Cho SJ, Chang SM, et al. Development and validation of the National Cancer Center Psychological Symptom Inventory. Psychooncology. 2017;26(7):1036-43.

16. EuroQol G. EuroQol-a new facility for the measurement of health-related quality of life. Health Policy. 1990;16(3):199-208.

17. Kim S, Won CW, Kim BS, Kim S, Yoo J, Byun S, et al. EuroQol Visual Analogue Scale (EQ-VAS) as a Predicting Tool for Frailty in Older Korean Adults: The Korean Frailty an Aging Cohort Study (KFACS). J Nutr Health Aging. 2018;22(10):1275-80.

18. Xiao W, Chow KM, So WK, Leung DY, Chan CW. The Effectiveness of Psychoeducational Intervention on Managing Symptom Clusters in Patients With Cancer: A Systematic Review of Randomized 
Controlled Trials. Cancer Nurs. 2016;39(4):279-91.

19. Wenzel LB, Fairclough DL, Brady MJ, Cella D, Garrett KM, Kluhsman BC, et al. Age-related differences in the quality of life of breast carcinoma patients after treatment. Cancer. 1999;86(9):1768-74.

20. Carlson LE, Angen M, Cullum J, Goodey E, Koopmans J, Lamont L, et al. High levels of untreated distress and fatigue in cancer patients. Br J Cancer. 2004;90(12):2297-304.

21. Koch $L$, Jansen $L$, Brenner $H$, Arndt $V$. Fear of recurrence and disease progression in long-term $(>/=5$ years) cancer survivors-a systematic review of quantitative studies. Psychooncology. 2013;22(1):111.

22. Moye J, June A, Martin LA, Gosian J, Herman LI, Naik AD. Pain is prevalent and persisting in cancer survivors: differential factors across age groups. J Geriatr Oncol. 2014;5(2):190-6.

23. Hodgkinson K, Butow P, Hunt GE, Pendlebury S, Hobbs KM, Wain G. Breast cancer survivors' supportive care needs 2-10 years after diagnosis. Support Care Cancer. 2007;15(5):515-23.

24. Gradishar WJ, Anderson BO, Balassanian R, Blair SL, Burstein HJ, Cyr A, et al. Breast Cancer, Version 4.2017, NCCN Clinical Practice Guidelines in Oncology. J Natl Compr Canc Netw. 2018;16(3):310-20.

25. Arimidex TAoiCTG, Forbes JF, Cuzick J, Buzdar A, Howell A, Tobias JS, et al. Effect of anastrozole and tamoxifen as adjuvant treatment for early-stage breast cancer: 100-month analysis of the ATAC trial. Lancet Oncol. 2008;9(1):45-53.

26. Ebede CC, Jang Y, Escalante CP. Cancer-Related Fatigue in Cancer Survivorship. Med Clin North Am. 2017;101(6):1085-97.

27. Minton O, Berger A, Barsevick A, Cramp F, Goedendorp M, Mitchell SA, et al. Cancer-related fatigue and its impact on functioning. Cancer. 2013;119(Suppl 11):2124-30.

28. Wolvers MDJ, Leensen MCJ, Groeneveld IF, Frings-Dresen MHW, De Boer A. Predictors for earlier return to work of cancer patients. J Cancer Surviv. 2018;12(2):169-77.

29. Lee MK, Kang HS, Lee KS, Lee ES. Three-Year Prospective Cohort Study of Factors Associated with Return to Work After Breast Cancer Diagnosis. J Occup Rehabil. 2017;27(4):547-58.

30. Stepanski EJ, Walker MS, Schwartzberg LS, Blakely LJ, Ong JC, Houts AC. The relation of trouble sleeping, depressed mood, pain, and fatigue in patients with cancer. J Clin Sleep Med. 2009;5(2):132-6.

31. Sharp L, O'Leary E, Kinnear H, Gavin A, Drummond FJ. Cancer-related symptoms predict psychological wellbeing among prostate cancer survivors: results from the PiCTure study. Psychooncology. 2016;25(3):282-91.

32. Glare PA, Davies PS, Finlay E, Gulati A, Lemanne D, Moryl N, et al. Pain in cancer survivors. J Clin Oncol. 2014;32(16):1739-47.

33. Savard J, Morin CM. Insomnia in the context of cancer: a review of a neglected problem. J Clin Oncol. 2001;19(3):895-908.

34. Savard J, Simard S, Ivers H, Morin CM. Randomized study on the efficacy of cognitive-behavioral therapy for insomnia secondary to breast cancer, part I: Sleep and psychological effects. J Clin 
Oncol. 2005;23(25):6083-96.

35. Oeffinger KC, Argenbright KE, Levitt GA, McCabe MS, Anderson PR, Berry E, et al. Models of cancer survivorship health care: moving forward. Am Soc Clin Oncol Educ Book. 2014.

doi:10.14694/EdBook_AM.2014.34.205.205-13.

36. Kinnane NA, Piper A, Wiley G, Nolte L, Evans J, Jefford M. Transforming Cancer Survivorship Care: An Australian Experience. Asia Pac J Oncol Nurs. 2017;4(2):91-4.

\section{Figures}

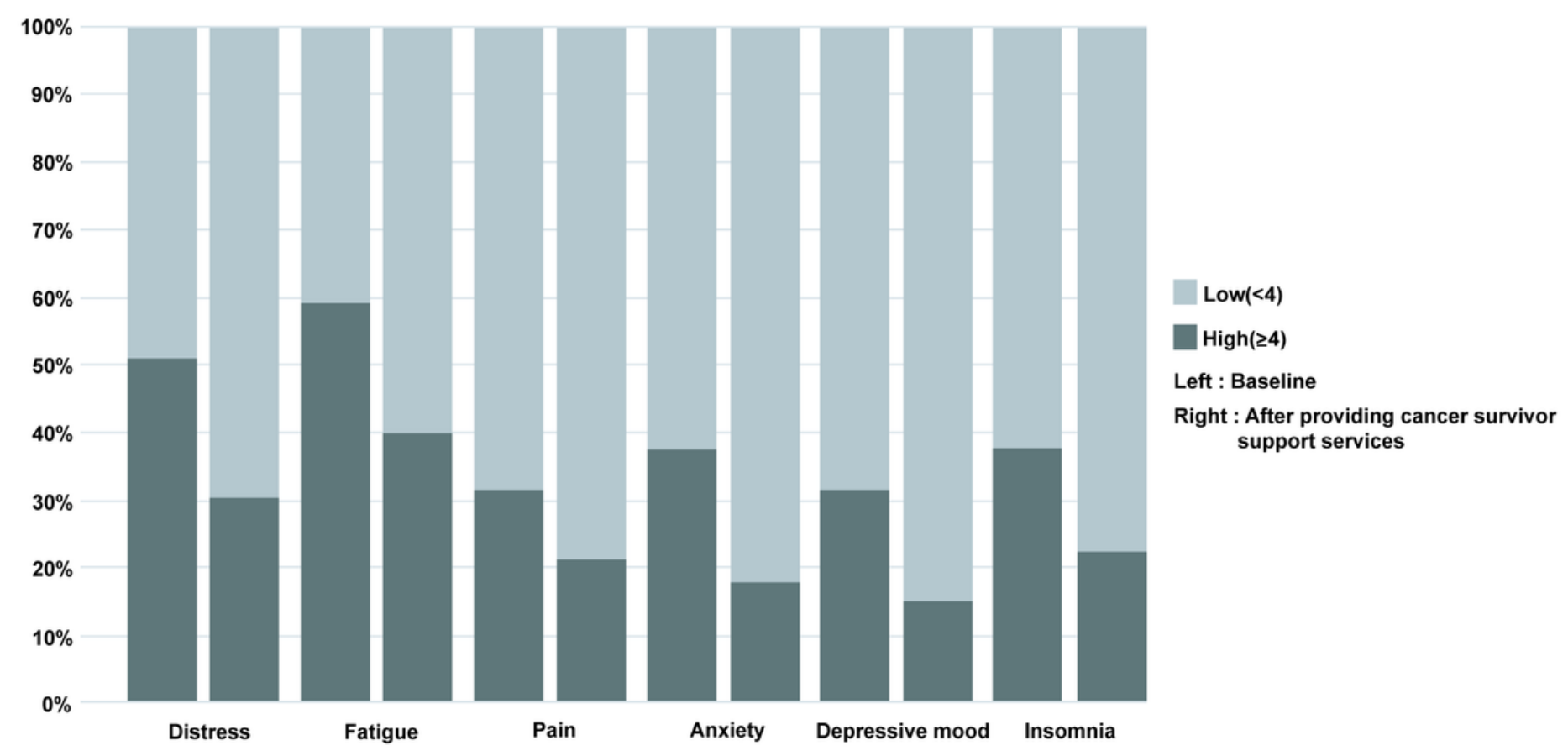

\section{Figure 1}

Changes in the proportion of high scores in distress, fatigue, pain, anxiety, depressive mood, and insomnia after providing support services for cancer survivors. A McNemar test was performed. All $p<$ 0.001 .

\section{Supplementary Files}

This is a list of supplementary files associated with this preprint. Click to download.

- SupplementarytableBMCCancer.docx 